\title{
Characterization of Uncertainty in Damping Modeling
}

\author{
Sondipon Adhikari $^{*}$ \\ University of Wales Swansea. Swansea, United Kingdom
}

\begin{abstract}
The characterization of uncertainty in the damping forces in a vibrating structure is of significant interest in structural dynamics. The most common approach is to use a viscous damping matrix with uncertain coefficients. The viscous damping is not the only damping model within the scope of linear analysis. Any model which makes the energy dissipation functional non-negative is a possible candidate for a valid damping model. There are many functional forms which can be used as damping models. In this paper two approaches are proposed to quantify such 'model-form uncertainty' associated with the use of the viscous damping model (not only the model parameters). The first approach is based on an ensemble of equivalent damping functions and the second approach is based on the random matrix theory. The results obtained from the two methods are compared using numerical examples. It was observed that the random matrix theory can be used to quantify damping model uncertainty in some cases.
\end{abstract}

\section{Nomenclature}

\begin{tabular}{|c|c|}
\hline $\mathbf{f}(t)$ & forcing vector \\
\hline $\mathbf{M}, \mathbf{C}$ and $\mathbf{K}$ & mass, damping and stiffness matrices respectively \\
\hline $\mathbf{u}(t)$ & response vector \\
\hline $\mathbf{Z}$ & a $n \times n$ symmetric complex matrix \\
\hline$\Gamma_{n}(a)$ & multivariate gamma function \\
\hline$\omega$ & excitation frequency \\
\hline$\phi_{(\bullet)}$ & characteristic function of $(\bullet)$ \\
\hline i & unit imaginary number, $\mathrm{i}=\sqrt{-1}$ \\
\hline$m, \Psi$ & scalar and matrix parameters of the inverted Wishart distribution \\
\hline$n$ & number of degrees of freedom \\
\hline$p, \Sigma$ & scalar and matrix parameters of the Wishart distribution \\
\hline \multicolumn{2}{|l|}{ Conventions } \\
\hline$(\bullet)^{T}$ & matrix transposition \\
\hline $\mathbb{R}$ & space of real numbers \\
\hline $\mathbb{R}_{n}^{+}$ & space $n \times n$ real positive definite matrices \\
\hline $\mathbb{R}^{n}$ & space $n \times 1$ real vector \\
\hline $\mathbb{R}^{n \times m}$ & space $n \times m$ real matrices \\
\hline$|\bullet|$ & determinant of a matrix \\
\hline $\operatorname{etr}\{\bullet\}$ & $\exp \{\operatorname{Trace}(\bullet)\}$ \\
\hline $\mathrm{E}[(\bullet)]$ & mathematical expectation operator \\
\hline$\otimes$ & Kronecker product $\left(\mathrm{sec}^{1}\right)$ \\
\hline$\sim$ & distributed as \\
\hline Trace $(\bullet)$ & sum of the diagonal elements of a matrix \\
\hline$p_{(\bullet)}(\mathbf{X})$ & probability density function of $(\bullet)$ in (matrix) variable $\mathbf{X}$ \\
\hline $\mathbb{L}\{(\bullet)\}$ & Laplace transform of $(\bullet)$ \\
\hline FRF & Frequency Response Function \\
\hline
\end{tabular}

* Chair of Aerospace Engineering School of Engineering University of Wales Swansea Singleton Park, Swansea SA2 8PP, UK, AIAA Senior Member.

Copyright (c) 2007 by the American Institute of Aeronautics and Astronautics, Inc. The U.S. Government has a royalty-free license to exercise all rights under the copyright claimed herein for Governmental purposes. All other rights are reserved by the copyright owner. 


\section{Introduction}

$\mathrm{U}$ NCERTAINTIES are unavoidable in the description of real-life engineering systems. The quantification of uncertainties plays a crucial role in establishing the credibility of a numerical model. Uncertainties can be broadly divided into two categories. The first type is due to the inherent variability in the system parameters. This type of uncertainty is often referred to as aleatoric uncertainty. If enough samples are present, it is possible to characterize the variability using well established statistical methods and consequently the probability density functions (pdf) of the parameters can be obtained. The second type of uncertainty is due to the lack of knowledge regarding a system, often referred to as epistemic uncertainty or model uncertainty. This kind of uncertainty generally arises in the modelling of complex physical phenomenon such as damping. Quantification of uncertainties associated with the damping forces is difficult because, unlike inertia and stiffness forces, it is not in general clear what are the state variables that govern the damping forces. The most common approach is to use the 'viscous damping' where the instantaneous generalized velocities are the only relevant state variables. Several studies exist where viscous damping coefficients are assumed to be random variables. However, considering randomness in the viscous damping parameters would not address the fundamental uncertainty that arises due to the use of viscous damping model itself. Viscous damping is by no means the only damping model within the scope of a linear analysis. Any model which makes the energy dissipation functional non-negative is a possible candidate for a valid damping model. Therefore, to avoid any 'model biases', in this study we have used possibly the most general linear damping model expressed by the following convolution integral

$$
\mathbf{F}_{d}(t)=\int_{-\infty}^{t} \mathcal{G}(t-\tau) \dot{\mathbf{u}}(\tau) \mathrm{d} \tau
$$

where $\mathbf{u}(t) \in \mathbb{R}^{n}$ is the vector of generalized coordinates with $t \in \mathbb{R}^{+}$denotes time, $\mathcal{G}(\hat{t}) \in \mathbb{R}^{n \times n}$ is the kernel function matrix and $\hat{t}$ is a generalized time. This model was used by $\operatorname{Biot}^{2}$ in the context of viscoelastic materials and later used by several authors ${ }^{3-10}$ in structural dynamics applications. In the special case when $\mathcal{G}(t-\tau)=\mathbf{C} \delta(t-\tau)$, where $\delta(t)$ is the Dirac-delta function, equation (1) reduces to the case of viscous damping.

The most common method to model damping in multiple-degree-of-freedom linear systems is to assume the so called viscous damping. Many researches have proposed methods to identify a viscous damping matrix from experimental measurements (see Pilkey and Inman $^{11}$ for a survey). Although these methods allow one to obtain the viscous damping matrix with a reasonable degree of confidence, they do not address the fundamental question whether the viscous damping model itself is the correct damping model to be used for a given system. This paper is devoted to quantify such 'model-form uncertainty' associated with the use of the viscous damping model.

The equation of motion of lumped parameter linear systems with $n$ degrees-of-freedom with general damping of the form (1) can be expressed as

$$
\mathbf{M u ̈}(t)+\int_{0}^{t} \mathcal{G}(t-\tau) \dot{\mathbf{u}}(\tau) \mathrm{d} \tau+\mathbf{K u}(t)=\mathbf{f}(t) .
$$

Here $\mathbf{f}(t) \in \mathbb{R}^{n}$ is the vector of applied forcing, $\mathbf{M} \in \mathbb{R}^{n \times n}$ is the mass matrix, $\mathbf{K} \in \mathbb{R}^{n \times n}$ is the stiffness matrix. The mass and the stiffness matrices are symmetric and positive definite matrices and can be obtained using the finite element method. ${ }^{12}$ It is in general difficult to obtain the matrix of the damping kernel functions from experimental measurements. Therefore, the uncertainty in damping arises from the functional form of the elements of $\mathcal{G}(\hat{t})$ and not only in the parameters of a particular function.

In this paper we are primarily interested in the frequency response function (FRF) of system (2). The previous study by Adhikari and Woodhouse ${ }^{13,14}$ shows that even when the non-viscous effect is strong, a viscous damping matrix with proper parameters may represent the FRF well provided the overall damping is light. Because in majority of aircraft and automotive structures the damping is light (typically less than 5\%), in this paper we consider the possibility of quantifying the uncertainty arising due to damping model-form using a random viscous damping matrix. More precisely, the idea is to quantify uncertainty arising due to different functional form in $\mathcal{G}(\hat{t})$ by a constant random matrix $\mathbf{C} \in \mathbb{R}^{n \times n}$. That is, we want to 'replace' equation (2) with uncertain functional forms in $\mathcal{G}(\hat{t})$ by a viscously damped system

$$
\mathbf{M} \ddot{\mathbf{u}}(t)+\mathbf{C} \dot{\mathbf{u}}(t)+\mathbf{K u}(t)=\mathbf{f}(t)
$$


with a random $\mathbf{C} \in \mathbb{R}^{n \times n}$ matrix. If a random viscous damping matrix $\mathbf{C}$ can indeed capture the damping model-form uncertainty, then it would significantly help the damping modelling process. This paper is specifically focused on the uncertainty in damping. But it should be remembered that there can be significant uncertainty in the elements of the mass and stiffness matrices also. The uncertainty in the mass and stiffness matrices can be usually treated independently from the damping and a complete discussion is beyond the scope of this paper. Readers are instead referred to the review papers ${ }^{15-17}$ for further discussions.

Two approaches are proposed and compared in this paper. The first approach is based on an ensemble of equivalent damping functions and the second approach is based on random matrix theory. In the first approach in section II different equivalent functional forms of $\mathcal{G}(\hat{t})$ are derived and their parameters are selected. The collection of these different equivalent functional forms are then assumed to form the random sample space. Under these settings, the selection of any one model (such as the viscous model) can be regarded as a random event in the sample space of the admissible functions. In the second approach in section III, the viscous damping matrix $\mathbf{C}$ is considered to be a random matrix whose distribution is obtained using the maximum entropy principle. ${ }^{18,19}$ The results obtained from the two methods are compared using numerical examples.

\section{Sample space of damping models}

It is well recognized that in general a physically realistic model of damping will not be viscous. Damping models in which the dissipative forces depend on any quantity other than the instantaneous generalized velocities are non-viscous damping models. Mathematically, any causal model which makes the energy dissipation functional non-negative is a possible candidate for a non-viscous damping model. Clearly a wide range of choice is possible, either based on the physics of the problem, or by a priori selecting a model and fitting its parameters from experiments.

Equation (1) is very general and for any engineering applications some specific form of $\mathcal{G}(\hat{t})$ have to be assumed. A wide variety of mathematical expressions could be used for the kernel functions $\mathcal{G}(\hat{t})$. A physically realistic damping kernel function must model dissipation of (vibration) energy in the system. This fact restricts the possible forms of the kernel functions which may be used. This condition implies that the rate of energy dissipation given by

$$
\mathcal{D}(t)=\frac{1}{2} \dot{\mathbf{u}}(t)^{T} \int_{-\infty}^{t} \mathcal{G}(t-\tau) \dot{\mathbf{u}}(\tau) \mathrm{d} \tau,
$$

should be non-negative. We consider the simple case in which just one damping kernel function is used. In that case the general form of the kernel function in equation (1) reduces to

$$
\mathcal{G}(\hat{t})=\mathbf{C} g(\hat{t})
$$

where $g(\hat{t})$ is some damping function and $\mathbf{C}$ is a non-negative definite coefficient matrix. The admissible form of $g(\hat{t})$ is restricted by the condition of non-negative energy loss given in equation (4). The damping model in equation (5) is physically realistic if the real part of the Fourier transform of the kernel function is non-negative within the driving frequency range, that is $\Re[G(\mathrm{i} \omega)] \geq 0, \forall \omega$. This can be easily shown. Rewriting equation (4) in the frequency domain and using (5), the rate of energy dissipation can be expressed as

$$
D(\mathrm{i} \omega)=\frac{\omega^{2}}{2} \Re\left\{\overline{\mathbf{u}}^{*^{T}} \mathbf{C} \overline{\mathbf{u}} G(\mathrm{i} \omega)\right\}
$$

where $\Re(\bullet)$ represents the real part of $(\bullet), G(\mathrm{i} \omega)$ and $\overline{\mathbf{u}}$ are the Fourier transforms of $g(t)$ and $\mathbf{u}(t)$ respectively. For a physically realistic model of damping we must have

$$
\begin{array}{ll} 
& D(\mathrm{i} \omega) \geq 0 \\
\text { or } \quad & \frac{\omega^{2}}{2} \Re\left\{\overline{\mathbf{u}}^{*^{T}} \mathbf{C} \overline{\mathbf{u}} G(\mathrm{i} \omega)\right\} \geq 0 \\
\text { or } \quad \Re\{G(\mathrm{i} \omega)\} \geq 0
\end{array}
$$

since for a real value of driving frequency $\omega^{2} \geq 0$ and $\Re\left\{\overline{\mathbf{u}}^{*^{T}} \mathbf{C} \overline{\mathbf{u}}\right\} \geq 0$ as $\mathbf{C}$ is a non-negative definite matrix. Some of the damping functions used in the literature are shown in Table 1. If multiple kernel functions are used, then we may have

$$
\mathcal{G}(\hat{t})=\sum_{j=1}^{n} \mathbf{C}_{(j)} g_{(j)}(\hat{t})
$$


Table 1. A summary of non-viscous damping functions in the Laplace domain where the Laplace variable $s=\mathrm{i} \omega$.

\begin{tabular}{|c|c|c|}
\hline $\begin{array}{l}\text { Model } \\
\text { Number }\end{array}$ & Damping function & Author and year of publication \\
\hline 1 & $G(s)=\sum_{k=1}^{n} \frac{a_{k} s}{s+b_{k}}$ & Biot $^{20}-1955$ \\
\hline 2 & $G(s)=\frac{E_{1} s^{\alpha}-E_{0} b s^{\beta}}{1+b s^{\beta}} \quad \begin{array}{c}(0<\alpha, \beta<1) \\
s^{2}+2 \xi_{k} \omega_{k} s\end{array}$ & Bagley and Torvik ${ }^{21}-1983$ \\
\hline 3 & $s G(s)=G^{\infty}\left[1+\sum_{k} \alpha_{k} \frac{s^{2}+2 \xi_{k} \omega_{k} s}{s^{2}+2 \xi_{k} \omega_{k} s+\omega_{k}^{2}}\right]$ & $\begin{array}{l}\text { Golla and Hughes }^{22}-1985 \\
\text { and McTavish and Hughes }{ }^{23}-1993\end{array}$ \\
\hline 4 & $G(s)=1+\sum_{k=1}^{n} \frac{\Delta_{k} s}{s+\beta_{k}}$ & Lesieutre and Mingori ${ }^{24}-1990$ \\
\hline 5 & $G(s)=c \frac{1-e^{-s t_{0}}}{s t_{0}}$ & Adhikarii $^{25}-1998$ \\
\hline 6 & $G(s)=\frac{c}{s t_{0}} \frac{1+2\left(s t_{0} / \pi\right)^{2}-e^{-s t_{0}}}{1+2\left(s t_{0} / \pi\right)^{2}}$ & Adhikari $^{25}-1998$ \\
\hline 7 & $G(s)=c e^{s^{2} / 4 \mu}\left[1-\operatorname{erf}\left(\frac{s}{2 \sqrt{\mu}}\right)\right]$ & Adhikari and Woodhouse ${ }^{13}-2001$ \\
\hline
\end{tabular}

where $n$ denotes the number of different kernel functions used in the study. Rewriting equation (8) in the frequency domain we have

$$
\mathbf{G}(\mathrm{i} \omega)=\sum_{k=1}^{n} \mathbf{C}_{(j)} G_{(j)}(\mathrm{i} \omega)
$$

where $\mathbf{G}(\mathrm{i} \omega)$ and $G_{(j)}(\mathrm{i} \omega)$ are the Fourier transforms of $\mathcal{G}(\hat{t})$ and $g_{(j)}(\hat{t})$ respectively. Extending the analysis proposed here we can say that a sufficient condition for equation (8) to be a valid damping model is $\Re\left\{G_{(j)}(\mathrm{i} \omega)\right\} \geq 0, \forall j$. From this discussion we therefore have the following general condition:

Condition: A sufficient condition for a physically realistic damping model is that the real part of the Fourier transform of the damping kernel function matrix is non-negative definite within the driving frequency range, that is $\Re[\mathbf{G}(\mathrm{i} \omega)] \geq$ $0, \forall \omega$.

\section{A. First-order equivalent damping models}

The condition derived in the previous subsection completely defines the sample space of the damping matrices in linear structural dynamics. Clearly many (possibly uncountable in number) functions would satisfy this condition. Here we consider following eight types of kernel functions which belong to the sample space of the admissible damping functions:

MODEL 1: Exponential model

$$
g_{(1)}(\hat{t})=\mu_{1} \exp \left[-\mu_{1} \hat{t}\right] ; \quad G_{(1)}(s)=\frac{\mu_{1}}{s+\mu_{1}} .
$$

Model 2: Gaussian model

$$
g_{(2)}(\hat{t})=2 \sqrt{\frac{\mu_{2}}{\pi}} \exp \left[-\mu_{2} \hat{t}^{2}\right] ; \quad G_{(2)}(s)=e^{s^{2} / 4 \mu_{2}}\left[1-\operatorname{erf}\left(\frac{s}{2 \sqrt{\mu_{2}}}\right)\right] .
$$

MOdEL 3: Step function model

$$
g_{(3)}(\hat{t})=\left\{\begin{array}{ll}
1 / \mu_{3} & \left(0<\hat{t}<\mu_{3}\right) \\
0 & \left(\hat{t}>\mu_{3}\right)
\end{array} \quad ; \quad G_{(3)}(s)=\frac{1-e^{-s \mu_{3}}}{s \mu_{3}} .\right.
$$


Model 4: Cosine model

$$
\begin{aligned}
g_{(4)}(\hat{t}) & =\left\{\begin{array}{cc}
\frac{1}{\mu_{4}}\left[1+\cos \left(\frac{\pi \hat{t}}{\mu_{4}}\right)\right] & \left(0<\hat{t}<\mu_{4}\right) \\
0 & \left(\hat{t}>\mu_{4}\right)
\end{array} ;\right. \\
G_{(4)}(s) & =\frac{1}{s \mu_{4}} \frac{1+2\left(s \mu_{4} / \pi\right)^{2}-e^{-s \mu_{4}}}{1+2\left(s \mu_{4} / \pi\right)^{2}} .
\end{aligned}
$$

ModELs 5-8: Multiple exponential models

$$
g_{(5, \cdots 8)}(\hat{t})=\sum_{j=1}^{m} \widetilde{\mu}_{j} \exp \left[-\widetilde{\mu}_{j} \hat{t}\right] ; \quad G_{(5, \cdots 8)}(s)=\sum_{j=1}^{m} \frac{\widetilde{\mu}_{j}}{s+\widetilde{\mu}_{j}} ; \quad m=2,4,8,16 .
$$

Here $\mu_{i}$ and $\widetilde{\mu}_{i} i=1, \cdots, 4$ are real and positive constants. All the functions have already been scaled so that they have unit area when integrated to infinity, that is $\int_{0}^{\infty} g_{(j)}(\hat{t}) \mathrm{d} \hat{t}=1$. This makes them directly comparable with the viscous model, in which the corresponding damping function would be a unit delta function, that is, $g_{(0)}(\hat{t})=\delta(\hat{t})$. For this case the coefficient matrix $\overline{\mathbf{C}}$ would be the usual damping matrix. However, this normalization still does not provide a convenient equivalence between the different damping models. In order to make the damping models 'nominally identical' we further normalize the damping functions to make any probabilistic equivalence between the models meaningful.

We define the characteristic time constant $\theta_{j}$ for each damping function, via the first moment of $g_{(j)}(\hat{t})$ as

$$
\theta_{j}=\int_{0}^{\infty} \hat{t} g_{(j)}(\hat{t}) \mathrm{d} \hat{t}
$$

The characterization of non-viscous damping using the moments of the damping kernel function was proposed by Adhikari and Woodhouse. ${ }^{26}$ For the eight damping models considered here, the evaluation of the integral in equation (15) results

$$
\begin{aligned}
\theta_{1} & =\frac{1}{\mu_{1}}, \quad \theta_{2}=\frac{1}{\sqrt{\pi \mu_{2}}}, \quad \theta_{3}=\frac{\mu_{3}}{2}, \quad \theta_{4}=\frac{\left(\pi^{2}-4\right) \mu_{4}}{2 \pi^{2}} \\
\text { and } \theta_{5, \cdots 8} & =\sum_{j=1}^{m} \frac{1}{\widetilde{\mu}_{j}} ; \quad m=2,4,8,16 .
\end{aligned}
$$

We call these damping models as the 'first-order equivalent damping models' because their first-order moments are equal. For the viscous damping clearly $\theta_{0}=0$. Therefore, the characteristic time constant of a damping function gives a convenient measure of 'width': if it is close to zero the damping behaviour will be near-viscous, and vice versa. To establish an equivalence between the eight damping models, we can choose the parameters so that they have the same time constant. Equating all $\theta_{j}$ in equation (16) one has

$$
\frac{1}{\mu_{1}}=\frac{1}{\sqrt{\pi \mu_{2}}}=\frac{\mu_{3}}{2}=\frac{\left(\pi^{2}-4\right) \mu_{4}}{2 \pi^{2}}=\sum_{j=1}^{m} \frac{1}{\widetilde{\mu}_{j}} .
$$

Because the first four models have only one parameter, this equation is sufficient to obtain the parameters uniquely. For multiple exponential models 5-8, we assume that the constants are random positive numbers such that equation (17) is satisfied. These eight models are plotted in figure 1 for two values of $\theta_{j}$. The damping kernel functions in figure 1(a) is closer to the viscous damping than the ones in figure 1 (b). Here these eight damping models are assumed to form the compete sample space of damping models. Clearly eight models are not enough to draw reliable statistical conclusions. The purpose of this paper is to introduce the concept of damping model sample space - should more damping models be envisaged, they can be included to expand the size of the sample space.

\section{Random damping matrix}

The main difficulty in the quantification of model-form uncertainty is that it is not possible to consider any uncertain parameters. This is because of the fact that there is no 'fixed function' and consequently no parameters to fit a probability density function. In this situation the non-parametric approach ${ }^{18,19,27}$ provided by the random matrix theory is useful. 


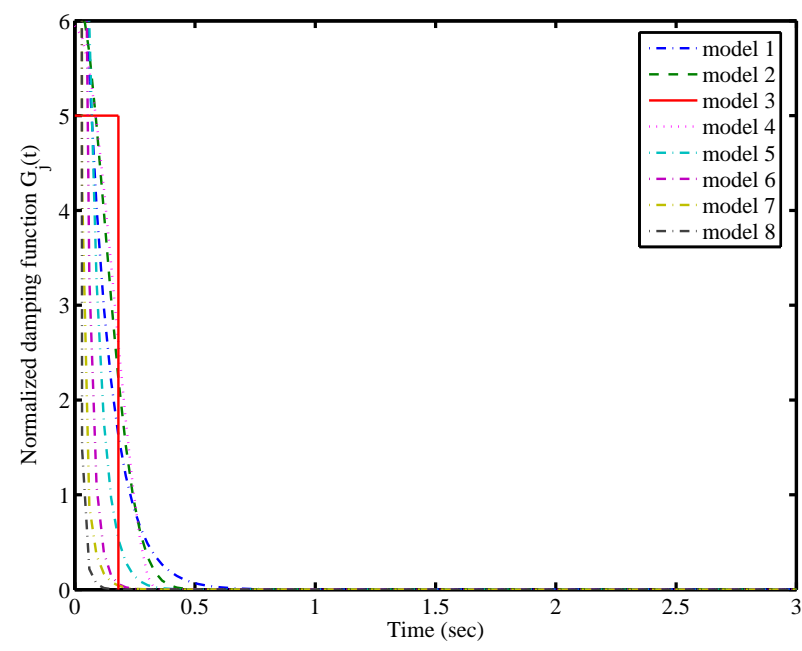

(a)

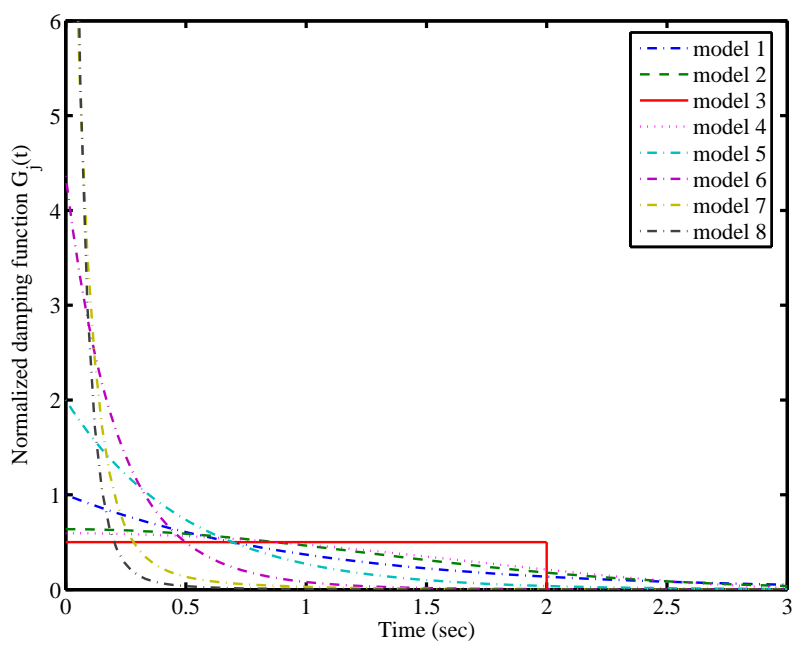

(b)

Figure 1. Eight models for the damping kernel functions; (a) $\theta_{j}=0.1$; (b) $\theta_{j}=1.0$.

\section{A. A brief review of matrix variate distributions}

Random matrices were introduced by Wishart ${ }^{28}$ during the late 1920 s in the context of multivariate statistics. However, Random Matrix Theory (RMT) was not used in other branches until 1950s when Wigner ${ }^{29}$ published his works on the eigenvalues of random matrices arising in high-energy physics. Using an asymptotic theory for large dimensional matrices, Wigner was able to bypass the Schrödinger equation and explain the statistics of measured atomic energy levels in terms of the limiting eigenvalues of these random matrices. Since then, the research on random matrices has continued to attract interests in multivariate statistics, physics, number theory and more recently in mechanical and electrical engineering. We refer the readers to the books by Mezzadri and Snaith, ${ }^{30}$ Tulino and Verdú, ${ }^{31}$ Eaton, ${ }^{32}$ Muirhead $^{33}$ and Mehta ${ }^{34}$ for the history and applications of the random matrix theory.

The probability density function of a random matrix can be defined in a manner similar to that of a random variable or random vector. If $\mathbf{A}$ is a $n \times m$ real random matrix, the matrix variate probability density function of $\mathbf{A} \in \mathbb{R}^{n \times m}$, denoted as $p_{\mathbf{A}}(\mathbf{A})$, is a mapping from the space of $n \times m$ real matrices to the real line, i.e., $p_{\mathbf{A}}(\mathbf{A}): \mathbb{R}^{n \times m} \rightarrow \mathbb{R}$. Here we define four types of random matrices which are relevant to probabilistic structural dynamics.

Definition 1. Gaussian random matrix: The random matrix $\mathbf{X} \in \mathbb{R}_{n, p}$ is said to have a matrix variate Gaussian distribution with a mean matrix $\mathcal{M} \in \mathbb{R}_{n, p}$ and a covariance matrix $\boldsymbol{\Sigma} \otimes \boldsymbol{\Psi}$, where $\boldsymbol{\Sigma} \in \mathbb{R}_{n}^{+}$and $\boldsymbol{\Psi} \in \mathbb{R}_{p}^{+}$provided the pdf of $\mathbf{X}$ is given by

$$
p_{\mathbf{X}}(\mathbf{X})=(2 \pi)^{-n p / 2}|\boldsymbol{\Sigma}|^{-p / 2}|\boldsymbol{\Psi}|^{-n / 2} \operatorname{etr}\left\{-\frac{1}{2} \boldsymbol{\Sigma}^{-1}(\mathbf{X}-\mathcal{M}) \Psi^{-1}(\mathbf{X}-\mathcal{M})^{T}\right\} .
$$

This distribution is usually denoted as $\mathbf{X} \sim N_{n, p}(\mathcal{M}, \mathbf{\Sigma} \otimes \mathbf{\Psi})$.

Definition 2. Wishart matrix: A $n \times n$ symmetric positive definite random matrix $\mathbf{S}$ is said to have a Wishart distribution with parameters $p \geq n$ and $\boldsymbol{\Sigma} \in \mathbb{R}_{n}^{+}$, if its $p d f$ is given by

$$
p_{\mathbf{S}}(\mathbf{S})=\left\{2^{\frac{1}{2} n p} \Gamma_{n}\left(\frac{1}{2} p\right)|\boldsymbol{\Sigma}|^{\frac{1}{2} p}\right\}^{-1}|\mathbf{S}|^{\frac{1}{2}(p-n-1)} \operatorname{etr}\left\{-\frac{1}{2} \boldsymbol{\Sigma}^{-1} \mathbf{S}\right\} .
$$

This distribution is usually denoted as $\mathbf{S} \sim W_{n}(p, \mathbf{\Sigma})$.

Definition 3. Matrix variate gamma distribution: A $n \times n$ symmetric positive definite random matrix $\mathbf{W}$ is said to have a matrix variate gamma distribution with parameters a and $\Psi \in \mathbb{R}_{n}^{+}$, if its pdf is given by

$$
p_{\mathbf{W}}(\mathbf{W})=\left\{\Gamma_{n}(a)|\boldsymbol{\Psi}|^{-a}\right\}^{-1}|\mathbf{W}|^{a-\frac{1}{2}(n+1)} \operatorname{etr}\{-\mathbf{\Psi} \mathbf{W}\} ; \quad \Re(a)>\frac{1}{2}(n-1) .
$$

This distribution is usually denoted as $\mathbf{W} \sim G_{n}(a, \mathbf{\Psi})$. The matrix variate gamma distribution was used by Soize $e^{18,19}$ for the random system matrices of linear dynamical systems. 
Definition 4. Inverted Wishart matrix: A $n \times n$ symmetric positive definite random matrix $\mathbf{V}$ is said to have an inverted Wishart distribution with parameters $m$ and $\Psi \in \mathbb{R}_{n}^{+}$, if its pdf is given by

$$
p_{\mathbf{V}}(\mathbf{V})=\frac{2^{-\frac{1}{2}(m-n-1) n|\boldsymbol{\Psi}|^{\frac{1}{2}(m-n-1)}}}{\Gamma_{n}\left(\frac{1}{2}(m-n-1)\right)|\mathbf{V}|^{m / 2}} \operatorname{etr}\left\{-\mathbf{V}^{-1} \boldsymbol{\Psi}\right\} ; \quad m>2 n, \boldsymbol{\Psi}>0
$$

This distribution is usually denoted as $\mathbf{V} \sim I W_{n}(m, \mathbf{\Psi})$.

In Eqs. (19)-(21), the function $\Gamma_{n}(a)$ is the multivariate gamma function, which can be expressed in terms of products of the univariate gamma functions as

$$
\Gamma_{n}(a)=\pi^{\frac{1}{4} n(n-1)} \prod_{k=1}^{n} \Gamma\left[a-\frac{1}{2}(k-1)\right] ; \quad \text { for } \quad \Re(a)>\frac{1}{2}(n-1) .
$$

The multivariate gamma function plays a key role in the random matrix method proposed in this paper. See Appendix A for a proof of (22) and related mathematical methods. For more details on the matrix variate distributions we refer to the books by Tulino and Verdú, ${ }^{31}$ Gupta and Nagar, ${ }^{35}$ Eaton, ${ }^{32}$ Muirhead ${ }^{33}$ and references therein. Among the four types of random matrices introduced above, the distributions given by Eqs. (19)-(21) always result in symmetric and non-negative definite matrices. Therefore, they can be possible candidates to model the random system matrices arising in probabilistic structural mechanics.

\section{B. Wishart model for the random damping matrix}

In this section an information theoretic approach is taken to obtain the matrix variate distribution of the random viscous damping matrix $\mathbf{C}$. First we look at the information available to us and then consider the constraints the matrix variate distribution must satisfy in order to be physically realistic. Once these steps are completed, the matrix variate distribution will be obtained using the maximum entropy method. In a series of papers Soize ${ }^{18,19}$ used this approach to obtain the probability density function of the system matrices.

Suppose that the mean of $\mathbf{C}$ is given by $\overline{\mathbf{C}}$. This information is likely to be available, for example, using the deterministic finite element method or experimental modal identification method. However, there are uncertainties associated with the damping model so that $\mathbf{C}$ is actually a random matrix. The distribution of $\mathbf{C}$ should be such that it is (a) symmetric, and (b) non-negative definite. Suppose the matrix variate density function of $\mathbf{C} \in \mathbb{R}^{n \times n}$ is given by $p_{\mathbf{C}}(\mathbf{C}): \mathbb{R}^{n \times n} \rightarrow \mathbb{R}$. We therefore have the following information and constrains to obtain $p_{\mathbf{C}}(\mathbf{C})$ :

$$
\begin{gathered}
\int_{\mathbf{C}>0} p_{\mathbf{C}}(\mathbf{C}) d \mathbf{C}=1 \quad \text { (normalization) } \\
\text { and } \mathrm{E}[\mathbf{C}]=\int_{\mathbf{C}>0} \mathbf{C} p_{\mathbf{C}}(\mathbf{C}) d \mathbf{C}=\overline{\mathbf{C}} \quad \text { (the mean matrix). }
\end{gathered}
$$

The mean matrix $\overline{\mathbf{C}}$ is symmetric and non-negative definite and the integrals appearing in these equations are $n(n+$ 1) $/ 2$ dimensional. The maximum entropy method ${ }^{36}$ can be used to obtain the probability density function of the random system matrices. Udwadia ${ }^{37,38}$ used an entropy based method to obtain the probability density functions of the mass, stiffness and damping constants of a single-degree-of-freedom oscillator. For multiple-degree-of-freedom systems, using the maximum entropy method Soize ${ }^{18,19}$ obtained the matrix variate gamma distribution for the system matrices given by Eq. (20). Note that the damping matrix needs to be positive definite only. There is no need for it to be invertible as long as the moments of the frequency response function (FRF) matrix

$$
\mathbf{H}(\mathrm{i} \omega)=\mathbf{D}^{-1}(\omega)=\left[-\omega^{2} \mathbf{M}+i \omega \mathbf{C}+\mathbf{K}\right]^{-1}
$$

exist.

In order to extend the maximum entropy method to random matrices, first note that the entropy associated with the matrix variate probability density function $p_{\mathbf{C}}(\mathbf{C})$ can be expressed as

$$
\mathcal{S}\left(p_{\mathbf{C}}\right)=-\int_{\mathbf{C}>0} p_{\mathbf{C}}(\mathbf{C}) \ln \left\{p_{\mathbf{C}}(\mathbf{C})\right\} d \mathbf{C} .
$$


Using this, together with the constrains in Eqs. (23) and (24) we construct the Lagrangian ${ }^{36}$

$$
\begin{aligned}
\mathcal{L}\left(p_{\mathbf{C}}\right)=-\int_{\mathbf{C}>0} p_{\mathbf{C}}(\mathbf{C}) \ln \left\{p_{\mathbf{C}}(\mathbf{C})\right\} d \mathbf{C}-\left(\lambda_{0}-1\right)\left(\int_{\mathbf{C}>0} p_{\mathbf{C}}(\mathbf{C}) d \mathbf{C}-1\right) & \\
& -\operatorname{Trace}\left(\boldsymbol{\Lambda}_{1}\left[\int_{\mathbf{C}>0} \mathbf{C}_{\mathbf{C}}(\mathbf{C}) d \mathbf{C}-\overline{\mathbf{C}}\right]\right) .
\end{aligned}
$$

The scalar $\lambda_{0} \in \mathbb{R}$ and the symmetric matrix $\Lambda_{1} \in \mathbb{R}^{n \times n}$ are the unknown Lagrange multiplies which need to be determined. Using the variational calculus it can be shown that the optimal condition is given by

$$
\begin{array}{ll} 
& \frac{\partial \mathcal{L}\left(p_{\mathbf{C}}\right)}{\partial p_{\mathbf{C}}}=0 \\
\text { or } \quad-\left(1+\ln \left\{p_{\mathbf{C}}(\mathbf{C})\right\}\right)-\left(\lambda_{0}-1\right)-\operatorname{Trace}\left(\boldsymbol{\Lambda}_{1} \mathbf{C}\right)=0 \\
\text { or } \quad-\ln \left\{p_{\mathbf{C}}(\mathbf{C})\right\}=\lambda_{0}+\operatorname{Trace}\left(\boldsymbol{\Lambda}_{1} \mathbf{C}\right) \\
\text { or } \quad p_{\mathbf{C}}(\mathbf{C})=\exp \left\{-\lambda_{0}\right\} \operatorname{etr}\left\{-\boldsymbol{\Lambda}_{1} \mathbf{C}\right\} .
\end{array}
$$

Using the matrix calculus, ${ }^{39-42}$ the Lagrange multipliers $\lambda_{0}$ and $\boldsymbol{\Lambda}_{1}$ can be obtained exactly by substituting $p_{\mathbf{C}}(\mathbf{C})$ from Eq. (31) into the constraint equations (23) and (24). After some algebra (see Appendix B for the details) it can be shown that

$$
p_{\mathbf{C}}(\mathbf{C})=\frac{r^{n r}|\overline{\mathbf{C}}|^{-r}}{\Gamma_{n}(r)} \operatorname{etr}\left\{-r \overline{\mathbf{C}}^{-1} \mathbf{C}\right\}, \quad \text { where } \quad r=\frac{1}{2}(n+1) .
$$

This distribution can be viewed as the matrix variate generalization of the single-degree-of-freedom case. ${ }^{37}$ If we consider the special case when $\mathbf{C}$ is an one dimensional $(n=1)$ matrix (that is a scalar, say $C$ ), then from Eq. (32) we obtain $p_{C}(C)=\exp (-C / \bar{C}) / \bar{C}$. This implies that $C$ becomes an exponentially distributed random variable which is well known ${ }^{36}$ that if we know only the mean of a random variable, then the maximum entropy distribution of that random variable becomes exponential. Therefore, the distribution in Eq. (32) can be viewed as the matrix generalization of the familiar exponential distribution.

Comparing Eq. (32) with the Wishart distribution in Eq. (19) it can be shown (see Eqs. (B.11) and (B.12) for the details) that $\mathbf{C}$ has the Wishart distribution with parameters $p=n+1$ and $\boldsymbol{\Sigma}=\overline{\mathbf{C}} /(n+1)$. Therefore, we have the following fundamental result regarding the damping modelling of linear structural dynamic systems:

Theorem 1. If only the mean of the damping matrix is available, say $\overline{\mathbf{C}}$, then the maximum-entropy $p d f$ of $\mathbf{C}$ follows the Wishart distribution with parameters $(n+1)$ and $\overline{\mathbf{C}} /(n+1)$, that is $\mathbf{C} \sim W_{n}(n+1, \overline{\mathbf{C}} /(n+1))$.

This distribution is the maximally uncertain distribution of the damping matrix. Next we investigate whether the Wishart distribution can be used quantify damping model uncertainty.

\section{Numerical example}

There is a major difference in emphasis between this study and other related studies on uncertainty in damping reported in the literature. Most of the reported analysis assume from the outset that the system is viscously damped and then characterize the uncertainty in the dynamic response due to the uncertainty in the viscous damping parameters. Here, we wish to investigate how much one can achieve by considering a Wishart random matrix model for the viscous damping matrix when the actual system is non-viscously damped, as one must expect to be the case for most practical systems. It is far from clear in practice what kind of non-viscous damping behaviour a system might exhibit. We defer that question for the moment, and instead study by simulation a system which can have eight different non-viscous damping models as introduced in subsection $\mathrm{A}$. These damping models are considered to form a sample space as in any random event. It is true that eight samples are far from satisfactory for a reliable statistical ensemble. However, the aim of this study is to get an initial estimation of the uncertainty arising due to random damping models. If the approach gives promising results, it can be extended to include more damping models to obtain further realistic results.

A system consisting of a linear array of spring-mass oscillators and dampers. This simple system gives us a useful basis to carry out numerical investigations. Figure 2 shows the model systems with $n$ masses, each of mass $m_{u}$, are connected by springs of stiffness $k_{u}$. Certain of the masses of the system shown in figure 2(a) have dissipative elements connecting them to the ground. In this case the damping force depends only on the absolute motion of the 


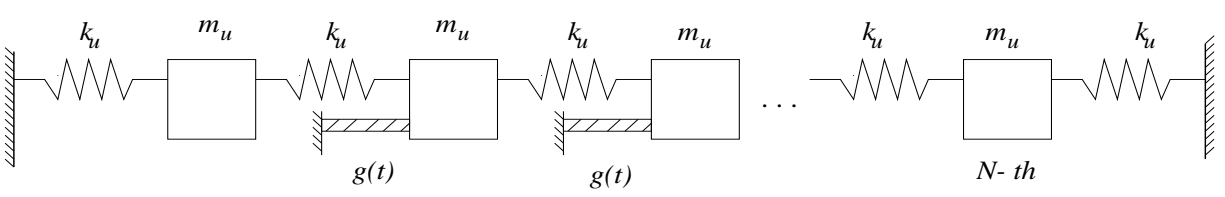

(a)

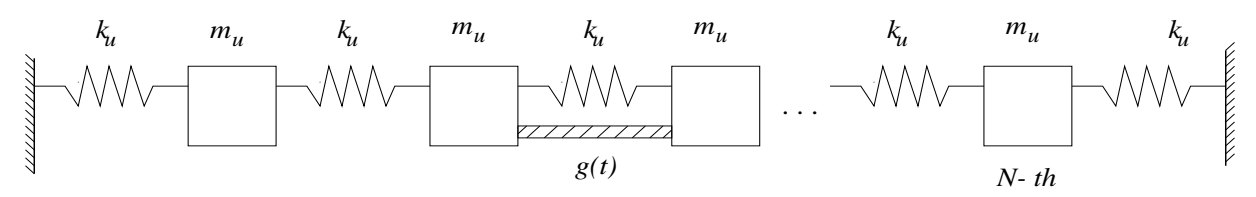

(b)

Figure 2. Linear array of $n$ spring-mass oscillators, $n=35, m_{u}=1 \mathrm{~kg}, k_{u}=4 \times 10^{3} \mathrm{~N} / \mathrm{m}$; (a) locally reacting damping; (b) non-locally reacting damping

individual masses. Such damping will be described as 'locally reacting'. For the system shown in figure 2(b), by contrast, dissipative elements are connected between certain adjacent pairs of masses. In this case the damping force depends on the relative motion of the two adjacent masses, and will be called 'non-locally reacting'.

For the system with locally reacting damping shown in figure $2(a), \overline{\mathbf{C}}=c \overline{\mathbf{I}}$ where $c$ is a constant and $\overline{\mathbf{I}}$ is a block identity matrix which is non-zero only between the $s$-th and $(s+l)$-th entries along the diagonal, so that ' $s$ ' denotes the first damped mass and $(s+l)$ the last one. For the system with non-locally reacting damping shown in figure 2(b), $\overline{\mathbf{C}}$ has a similar pattern to the stiffness matrix, but non-zero only for terms relating to the block between $s$ and $(s+l)$. For the numerical calculations considered here, we have taken $n=35, s=6$ and $(s+l)=28$. For the purpose of numerical examples, the values $m_{u}=1 \mathrm{~kg}, k_{u}=4 \times 10^{5} \mathrm{~N} / \mathrm{m}$ have been used. The resulting undamped natural frequencies then range from near zero to approximately $200 \mathrm{~Hz}$. For damping models, the value $c=27 \mathrm{Ns} / \mathrm{m} \mathrm{has}$ been used, and various values of the time constant $\theta_{j}$ have been tested. These are conveniently expressed as a fraction of the period of the highest undamped natural frequency:

$$
\theta=\gamma T_{\min }
$$

When $\gamma$ is small compared with unity the damping behaviour can be expected to be essentially viscous, but when $\gamma$ is of the order unity non-viscous effects should become significant.

We are principally interested in the mean and standard deviation of the amplitude of the frequency response function. For numerical calculations frequency-range up to $160 \mathrm{~Hz}$ is selected in this study. Two vales of $\gamma$, one small and the other relatively large, are selected. The values of the real part of $G(\mathrm{i} \omega)$ within the frequency range considered are shown in figure 3 . Note that when $\gamma=1.0$, model 4 become physically unrealistic between $60-80 \mathrm{~Hz}$ and model 3 become physically unrealistic between $50-100 \mathrm{~Hz}$ as $\Re[\mathbf{G}(\mathrm{i} \omega)]$ become negative. We still keep these models in our ensemble as they represent 'modelling errors'. By taking the Fourier transform of equation (2) and recalling that $\mathbf{G}(\mathrm{i} \omega)=\overline{\mathbf{C}} G(\mathrm{i} \omega)$, the matrix of the frequency response function can be obtained as

$$
\mathbf{H}(\mathrm{i} \omega)=\left[-\omega^{2} \mathbf{M}+i \omega \overline{\mathbf{C}} G(\mathrm{i} \omega)+\mathbf{K}\right]^{-1}
$$

In the numerical simulation, we generate the samples of the model-forms by choosing the eight different expressions of $G(\mathrm{i} \omega)$ in the above equation. The set of $\mathbf{H}(\mathrm{i} \omega)$ matrices obtained in this way is used as the ensemble for statistical calculations in the next two subsections.

\section{A. Results for Small $\gamma$}

When $\gamma=0.1$ all damping models show near-viscous behaviour. First consider the system shown in figure 2(a) with locally reacting damping. Figure 4 shows the mean and standard deviation of the amplitude of the FRF obtained using the eight damping models. Both the driving-point-FRF at mass location 11 and cross FRF between masses 11 and 24 are shown in the figure. In the same figures, the mean and the standard deviation of the amplitude of the FRF obtained using the proposed Wishart damping matrix are also shown. The mean obtained from both the approaches agree well with each other. Because all the eight damping models show near-viscous behaviour, the standard deviation obtained from the ensemble of the damping models is quite small, especially in the low-frequency range. As a result, 


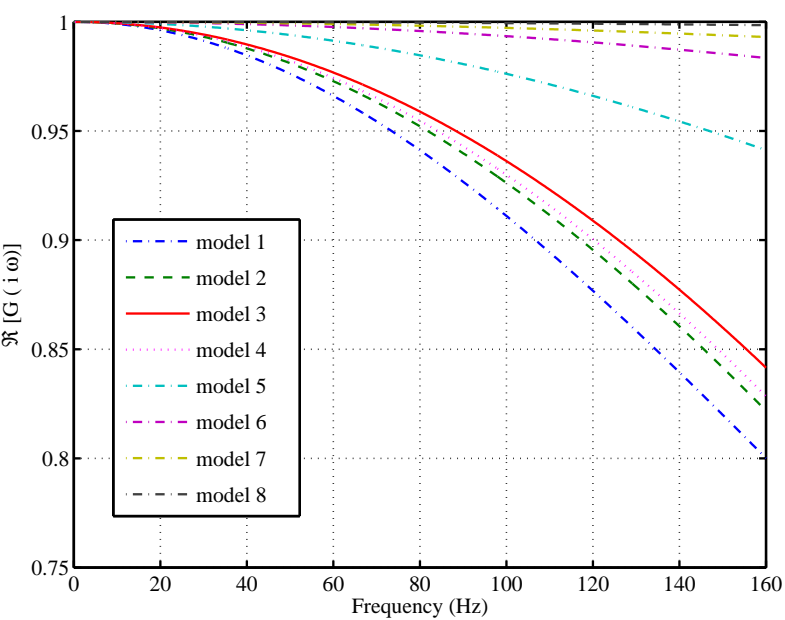

(a)

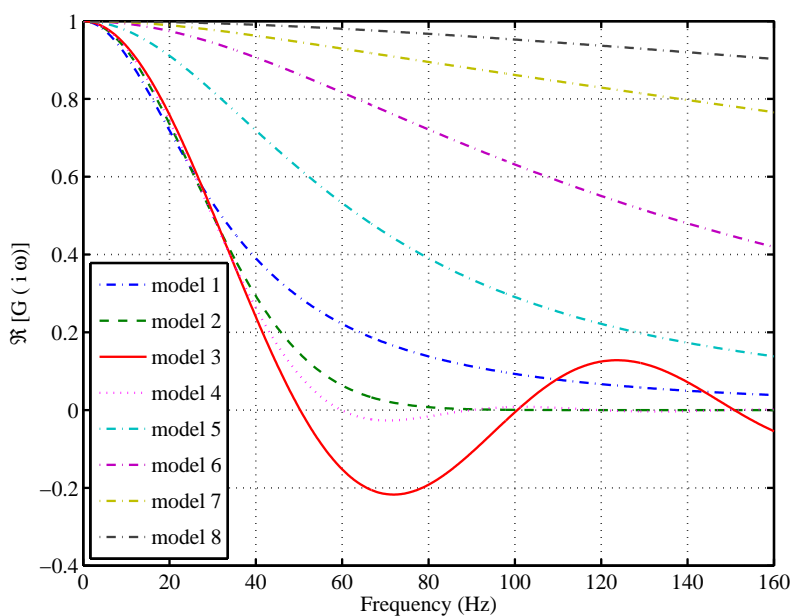

(b)

Figure 3. Real part of $G(\mathrm{i} \omega)$ for the eight damping models; (a) $\gamma=0.1$; (b) $\gamma=1.0$.

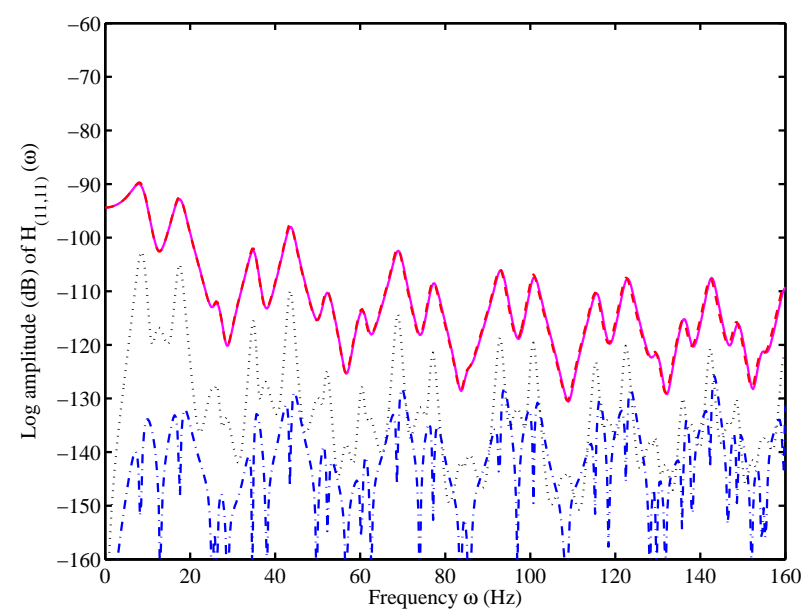

(a)

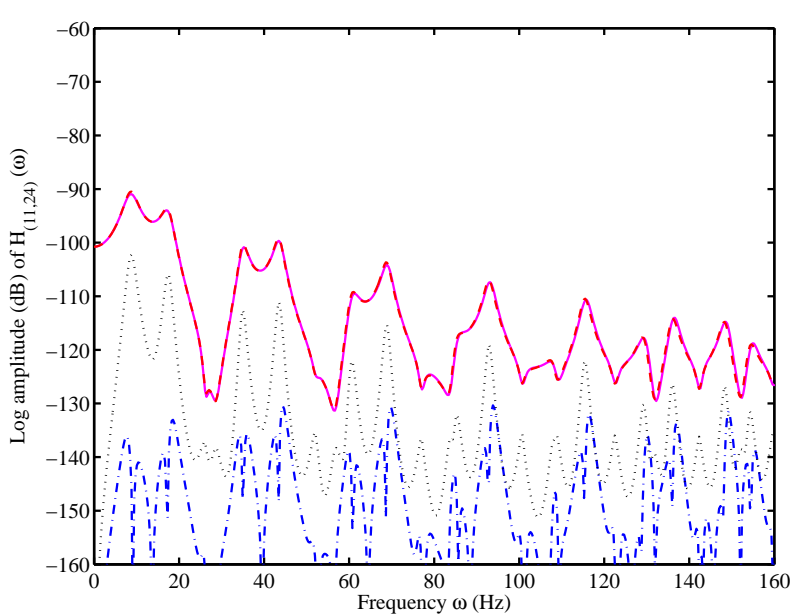

(b)

Figure 4. Comparison of the mean and standard deviation of the amplitude of the FRF obtained using eight damping models and proposed Wishart damping matrix; locally reacting damping, $\gamma=0.1$. - Ensemble average: Damping Models; - - Ensemble average: RMT; -. - . - Standard deviation: Damping Models; - .. Standard deviation: RMT; (a) driving-point-FRF; (b) cross-FRF

the standard deviation obtained from the Wishart damping matrix do not agree very well to that obtained from the ensemble of the damping models in the low-frequency range. However, there is a good agreement in the high frequency range.

Now we consider the system shown in figure 2 (b) with the alternative non-locally reacting damping. Figure 5 shows the mean and the standard deviation of the amplitude of the FRF obtained using the eight damping models. Both the driving-point-FRF and the cross FRF are shown in the figure. In the same figures, the mean and the standard deviation of the amplitude of the FRFs obtained using the proposed Wishart damping matrix are also shown. Like the previous case, the mean obtained from both the approaches agree well with each other. The standard deviation obtained from the Wishart damping matrix agree well to that obtained from the ensemble of damping models in the low-frequency range.

\section{B. Results for Larger $\gamma$}

When $\gamma$ is larger the non-viscous damping models depart from the viscous damping model, each in its own way. However, the average RMT results do not change with $\gamma$. We again consider the system shown in figure 2(a) with 


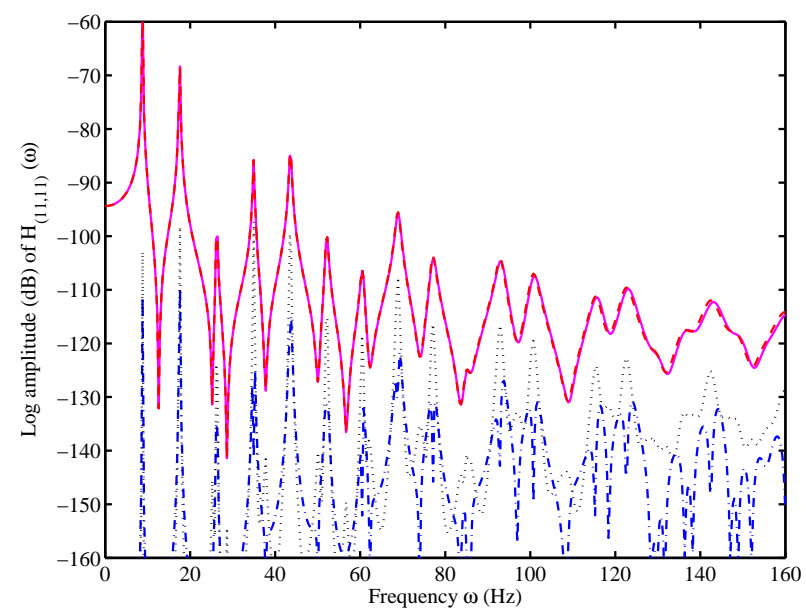

(a)

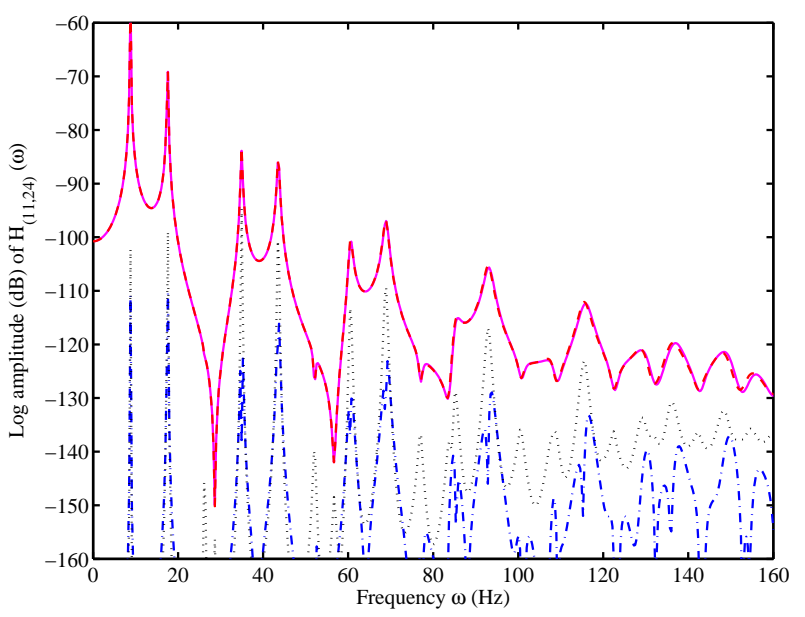

(b)

Figure 5. Comparison of the mean and standard deviation of the amplitude of the FRF obtained using eight damping models and proposed Wishart damping matrix; non-locally reacting damping, $\gamma=0.1$. - Ensemble average: Damping Models; -- Ensemble average: RMT; -. - .- Standard deviation: Damping Models; - . Standard deviation: RMT; (a) driving-point-FRF; (b) cross-FRF

locally reacting damping. Figure 6 shows the mean and the standard deviation of the amplitude of the FRF obtained using eight damping models. Both the driving-point-FRF at mass 11 and cross FRF between masses 11 and 24 are

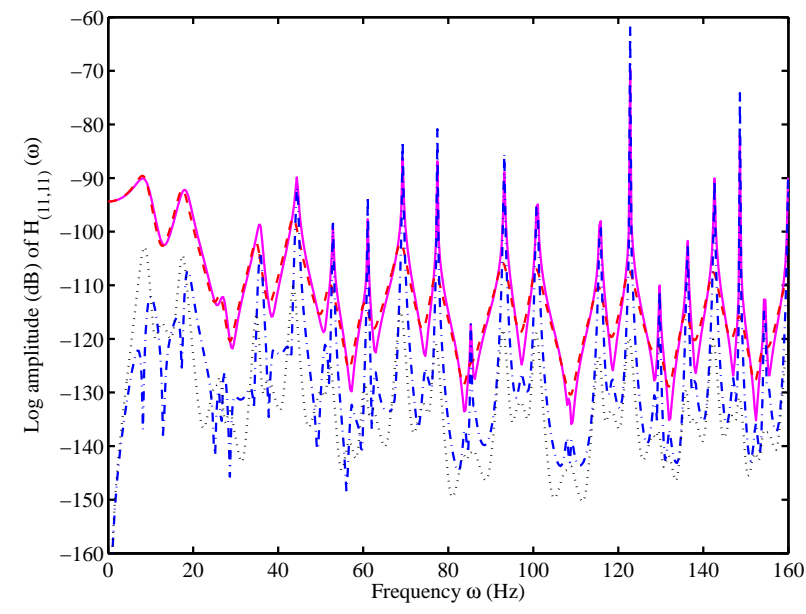

(a)

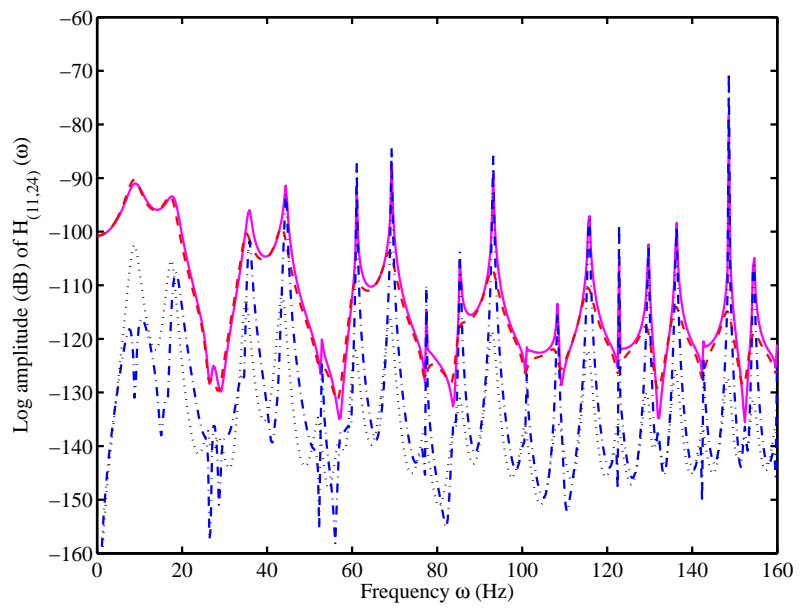

(b)

Figure 6. Comparison of the mean and standard deviation of the amplitude of the FRF obtained using eight damping models and proposed Wishart damping matrix; locally reacting damping, $\gamma=1.0$. - Ensemble average: Damping Models; -- Ensemble average: RMT; - . - .- Standard deviation: Damping Models; - . Standard deviation: RMT; (a) driving-point-FRF; (b) cross-FRF

shown in the figure. In the same figures, the mean and the standard deviation of the amplitude of the FRFs obtained using the proposed Wishart damping matrix are also shown. Unlike the case in the previous subsection, the mean obtained from both the approaches do not agree well in the high frequency range. The discrepancies are especially noticeable around the resonance frequencies. This is expected as damping affects a frequency response function only around the resonant frequencies. Because all the damping models depart from the viscous behaviour, the standard deviation obtained from the ensemble of the damping models is relatively higher, especially in the high-frequency range. The standard deviation obtained from the Wishart damping matrix agree well to that obtained from the ensemble of damping models across the frequency range.

Finally we consider the system shown in figure 2(b) with the alternative non-locally reacting damping. Figure 7 shows the mean and the standard deviation of the amplitude of the FRF obtained using the eight damping models. Both the driving-point-FRF and the cross FRF are shown in the figure. In the same figures, the mean and the standard 


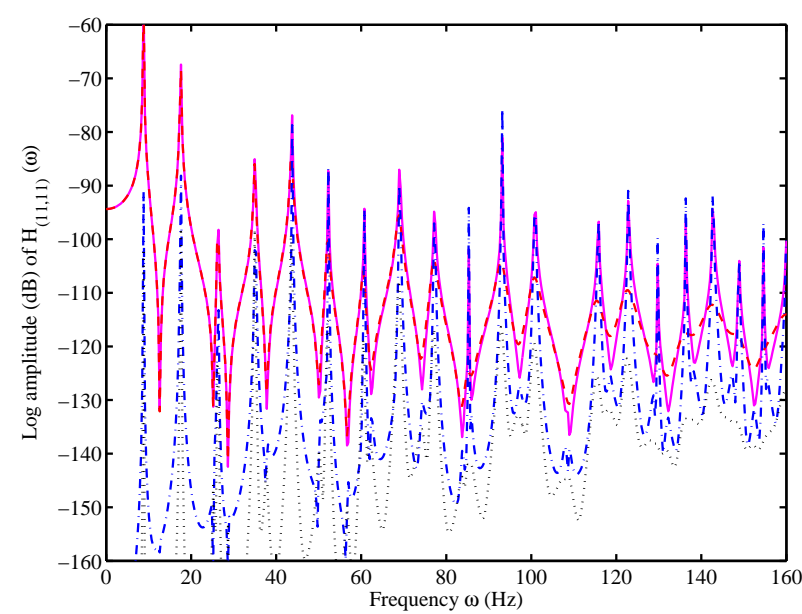

(a)

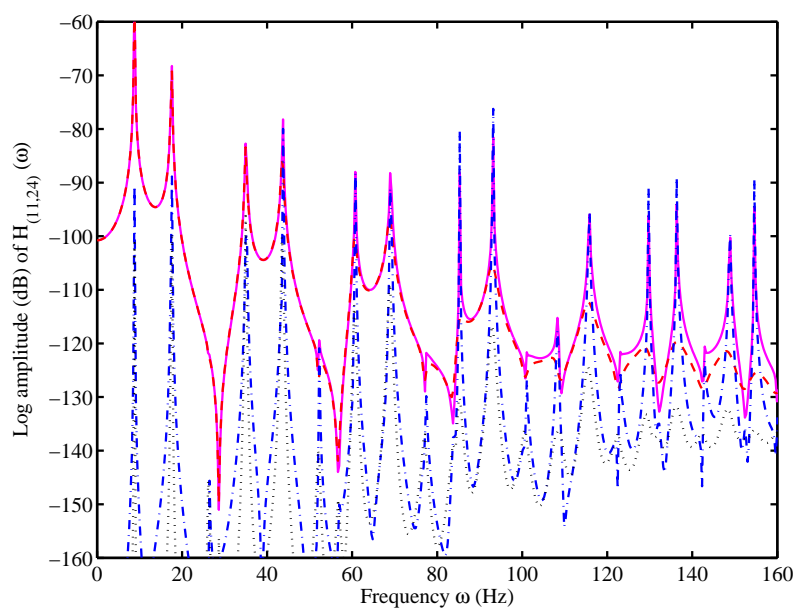

(b)

Figure 7. Comparison of the mean and standard deviation of the amplitude of the FRF obtained using eight damping models and proposed Wishart damping matrix; non-locally reacting damping, $\gamma=1.0$. - Ensemble average: Damping Models; -- Ensemble average: RMT; -. - .- Standard deviation: Damping Models; - . Standard deviation: RMT; (a) driving-point-FRF; (b) cross-FRF

deviation of the amplitude of the FRFs obtained using the proposed Wishart damping matrix are also shown. Unlike the case in the previous subsection, the mean obtained from both the approaches do not agree well in the high frequency range. However, the standard deviation obtained from the Wishart damping matrix agree well to that obtained from the ensemble of damping models in the low-frequency range. The agreement is acceptable in the high-frequency range also.

\section{Summary of results}

- If the damping functions are strongly non-viscous then the proposed Wishart random damping matrix can predict the model-form uncertainty reasonably well, especially in the low frequency range. The discrepancies in the high frequency range may be attributed to the fact that two out of eight damping models become non-physical in the higher frequency range.

- When the damping functions are near viscous then the proposed Wishart random damping matrix can predict the model-form uncertainty reasonably well, especially in the high frequency range. This is due to the fact that for the near-viscous functions, the standard deviation of the amplitude of the FRF is very small in the low-frequency region.

- The Wishart random matrix prediction of the model-form uncertainty is better for the non-locally damped system. The results for the four cases considered here are summarized in Table 2.

Table 2. A summary of the prediction of the model-form uncertainty using Wishart random damping matrix

\begin{tabular}{cll}
\hline \hline$\gamma$-values & Locally reacting dampers & Non-locally reacting dampers \\
\hline Low: $\gamma=0.1$ & $\begin{array}{l}\text { Good in the high-frequency range } \\
\text { (see figure 4) }\end{array}$ & $\begin{array}{l}\text { Good in the low-frequency range } \\
\text { (see figure 5) }\end{array}$ \\
High: $\gamma=1.0$ & $\begin{array}{l}\text { Acceptable across the low-frequency range } \\
\text { (see figure 6) }\end{array}$ & $\begin{array}{l}\text { Good across the low-frequency range } \\
\text { (see figure 7) }\end{array}$ \\
\hline \hline
\end{tabular}




\section{Conclusions and outlook}

The aim of this paper is to quantify uncertainty in damping modeling in linear dynamical systems. It is considered that in general the damping models are non-viscous and can be expressed by convolution integrals over some kernel functions. The condition the kernel functions must satisfy for the damping to be physically realistic has been derived. Two novel approaches to quantify uncertainty arising due to the possibility of different damping models have been proposed. The first approach is based on an ensemble of equivalent damping functions and the second approach is based on the random matrix theory. In the first approach, different equivalent functional forms of damping are derived and their parameters are selected using the new concept of 'the first-order equivalent damping models'. The collection of these different equivalent functional forms are then considered to constitute the random sample space so that the selection of any one the models (such as the viscous model) can be regarded as a random event in the space of these admissible functions. In the second approach, the viscous damping matrix is considered to be a random Wishart matrix. The results obtained from the two methods are compared using numerical examples.

Numerical experiments have been carried out with a wide range of parameter values and different damping models. The main features of the results have been illustrated by two representative parameter values. It was observed that If the damping functions are strongly non-viscous then the proposed Wishart random damping matrix can be used to predict the model-form uncertainty reasonably well in the low frequency region. Further research is needed to verify the applicability of these results to more complex systems.

\section{Acknowledgments}

The author acknowledges the support of the UK Engineering and Physical Sciences Research Council (EPSRC) through the award of an Advanced Research Fellowship, grant number GR/T03369/01.

\section{References}

${ }^{1}$ Graham, A., Kronecker Products and Matrix Calculus With Applications, Mathematics and its Applications, Ellis Horwood Limited, Chichester, UK, 1981

${ }^{2}$ Biot, M. A., "Linear thermodynamics and the mechanics of solids," Proceedings of the Third U. S. National Congress on Applied Mechanics, ASME, New York, 1958, pp. 1-18.

${ }^{3}$ Woodhouse, J., "Linear damping models for structural vibration," Journal of Sound and Vibration, Vol. 215, No. 3, 1998, pp. 547-569.

${ }^{4}$ Adhikari, S., "Dynamics of non-viscously damped linear systems," ASCE Journal of Engineering Mechanics, Vol. 128, No. 3, March 2002, pp. 328-339.

${ }^{5}$ Adhikari, S., "Eigenrelations for non-viscously damped systems," AIAA Journal, Vol. 39, No. 8, August 2001, pp. 1624-1630.

${ }^{6}$ Wagner, N. and Adhikari, S., "Symmetric state-space formulation for a class of non-viscously damped systems," AIAA Journal, Vol. 41, No. 5, 2003, pp. 951-956.

${ }^{7}$ Adhikari, S. and Wagner, N., "Analysis of asymmetric non-viscously damped linear dynamic systems," Transactions of ASME, Journal of Applied Mechanics, Vol. 70, No. 6, December 2003, pp. 885-893.

${ }^{8}$ Adhikari, S. and Wagner, N., "Direct time-domain approach for exponentially damped systems," Computer and Structures, Vol. 82, No. 29-30, November 2004, pp. 2453-2461.

${ }^{9}$ Adhikari, S., "Qualitative dynamic characteristics of a non-viscously damped oscillator," Proceedings of the Royal Society of London, SeriesA, Vol. 461, No. 2059, July 2005, pp. 2269-2288.

${ }^{10}$ Adhikari, S., "Dynamic response characteristics of a non-viscously damped oscillator," Transactions of ASME, Journal of Applied Mechanics, 2007, to appear.

${ }^{11}$ Pilkey, D. P. and Inman, D. J., "Survey of damping matrix identification," Proceedings of the 16th International Modal Analysis Conference (IMAC), Vol. 1, 1998, pp. 104-110.

${ }^{12}$ Zienkiewicz, O. C. and Taylor, R. L., The Finite Element Method, McGraw-Hill, London, 4th ed., 1991.

${ }^{13}$ Adhikari, S. and Woodhouse, J., "Identification of damping: part 1, viscous damping," Journal of Sound and Vibration, Vol. 243, No. 1, May 2001, pp. 43-61.

${ }^{14}$ Adhikari, S. and Woodhouse, J., "Identification of damping: part 2, non-viscous damping," Journal of Sound and Vibration, Vol. 243, No. 1, May 2001, pp. 63-88.

${ }^{15}$ Ibrahim, R. A., "Structural dynamics with parameter uncertainties," Applied Mechanics Reviews, ASME, Vol. 40, No. 3, 1987, pp. $309-328$.

${ }^{16}$ Benaroya, H., "Random eigenvalues, algebraic methods and structural dynamic models," Applied Mathematics and Computation, Vol. 52, 1992, pp. 37-66.

${ }^{17}$ Manohar, C. S. and Ibrahim, R. A., "Progress in structural dynamics with stochastic parameter variations: 1987 to 1998," Applied Mechanics Reviews, ASME, Vol. 52, No. 5, May 1999, pp. 177-197.

${ }^{18}$ Soize, C., "A nonparametric model of random uncertainties for reduced matrix models in structural dynamics," Probabilistic Engineering Mechanics, Vol. 15, No. 3, 2000, pp. 277-294.

${ }^{19}$ Soize, C., "A comprehensive overview of a non-parametric probabilistic approach of model uncertainties for predictive models in structural dynamics," Journal of Sound and Vibration, Vol. 288, No. 3, 2005, pp. 623-652. 
${ }^{20}$ Biot, M. A., "Variational principles in irreversible thermodynamics with application to viscoelasticity," Physical Review, Vol. 97, No. 6, 1955, pp. 1463-1469.

${ }^{21}$ Bagley, R. L. and Torvik, P. J., "Fractional calculus-a different approach to the analysis of viscoelastically damped structures," AIAA Journal, Vol. 21, No. 5, May 1983, pp. 741-748.

${ }^{22}$ Golla, D. F. and Hughes, P. C., "Dynamics of viscoelastic structures - a time domain finite element formulation," Transactions of ASME, Journal of Applied Mechanics, Vol. 52, December 1985, pp. 897-906.

${ }^{23}$ McTavish, D. J. and Hughes, P. C., "Modeling of linear viscoelastic space structures," Transactions of ASME, Journal of Vibration and Acoustics, Vol. 115, January 1993, pp. 103-110.

${ }^{24}$ Lesieutre, G. A. and Mingori, D. L., "Finite element modeling of frequency-dependent material properties using augmented thermodynamic fields," AIAA Journal of Guidance, Control and Dynamics, Vol. 13, 1990, pp. 1040-1050.

${ }^{25}$ Adhikari, S., Energy Dissipation in Vibrating Structures, Master's thesis, Cambridge University Engineering Department, Cambridge, UK, May 1998, First Year Report.

${ }^{26}$ Adhikari, S. and Woodhouse, J., "Quantification of non-viscous damping in discrete linear systems," Journal of Sound and Vibration, Vol. 260, No. 3, February 2003, pp. 499-518.

${ }^{27}$ Adhikari, S., "A non-parametric approach for uncertainty quantification in elastodynamics," 47th AIAA/ASME/ASCE/AHS/ASC Structures, Structural Dynamics \& Materials Conference, Newport, Rhode Island, USA, May 2006.

${ }^{28}$ Wishart, J., "The generalized product moment distribution in samples from a normal multivariate population," Biometrika, Vol. 20, No. A, 1928, pp. 32-52.

${ }^{29}$ Wigner, E. P., "On the Distribution of the Roots of Certain Symmetric Matrices," Annals of Mathematics, Vol. 67, No. 2, 1958 , pp. 325-327.

${ }^{30}$ Mezzadri, F. and Snaith, N. C., editors, Recent Perspectives in Random Matrix Theory and Number Theory, London Mathematical Society Lecture Note, Cambridge, U. K., 2005, Cambridge University Press.

${ }^{31}$ Tulino, A. M. and Verdú, S., Random Matrix Theory and Wireless Communications, now Publishers Inc., Hanover, MA, USA, 2004.

${ }^{32}$ Eaton, M. L., Multivariate Statistics: A Vector Space Approach, John Wiley \& Sons, New York, 1983.

${ }^{33}$ Muirhead, R. J., Aspects of Multivariate Statistical Theory, John Wiely and Sons, New York, USA, 1982.

${ }^{34}$ Mehta, M. L., Random Matrices, Academic Press, San Diego, CA, 2nd ed., 1991.

${ }^{35}$ Gupta, A. and Nagar, D., Matrix Variate Distributions, Monographs \& Surveys in Pure \& Applied Mathematics, Chapman \& Hall/CRC, London, 2000.

${ }^{36}$ Kapur, J. N. and Kesavan, H. K., Entropy Optimization Principles With Applications, Academic Press, San Diego, CA, 1992.

${ }^{37}$ Udwadia, F. E., "Response of Uncertain Dynamic-Systems .1," Applied Mathematics and Computation, Vol. 22, No. 2-3, 1987, pp. 115-150.

${ }^{38}$ Udwadia, F. E., "Response of Uncertain Dynamic-Systems .2," Applied Mathematics and Computation, Vol. 22, No. 2-3, 1987, pp. 151-187.

${ }^{39}$ Mathai, A. M., Jacobians of Matrix Transformation and Functions of Matrix Arguments, World Scientific, London, 1997.

${ }^{40}$ Steeb, W.-H., Matrix Calculus and the Kronecker Product with Applications and C++ Programs, World Scientific Publishing, London, 1997.

${ }^{41}$ Harville, D. A., Matrix Algebra from a Statistician's Perspective, Springer-Verlag, New York, 1998.

${ }^{42}$ Magnus, J. R. and Neudecker, H., Matrix Differential Calculus with Applications in Statistics and Econometrics, John Wiley \& Sons, New York, 1999.

${ }^{43}$ Gradshteyn, I. S. and Ryzhik, I. M., Table of Integrals, Series and Products, Academic Press, Inc., Boston, MA, USA, 5th ed., 1994, Tranlated from the Russian by Scripta Technica, Inc.

${ }^{44}$ Abramowitz, M. and Stegun, I. A., Handbook of Mathematical Functions, with Formulas, Graphs, and Mathematical Tables., Dover Publications, Inc., New York, USA, 1965.

${ }^{45}$ Kreyszig, E., Advanced engineering mathematics, John Wiley \& Sons, New York, nine ed., 2006.

\section{A. The Multivariate Gamma Function and Matrix Variate Laplace Transforms}

The multivariate gamma function $\Gamma_{n}(a)$ is defined as

$$
\Gamma_{n}(a)=\int_{\mathbf{X}>0} \operatorname{etr}\{-\mathbf{X}\}|\mathbf{X}|^{a-\frac{1}{2}(n+1)} d \mathbf{X} .
$$

Here $\Re(a)>\frac{1}{2}(n-1)$ and the integral in Eq. (A.1) is over the space of $n \times n$ symmetric positive definite matrices. Therefore, Eq. (A.1) represents a $n(n+1) / 2$ dimensional integral. Fortunately this integral can be evaluated exactly in a closed-form. ${ }^{35}$ This forms the basis of the analytical results given in the paper. Because $\mathbf{X}$ is a symmetric positive definite matrix, we can factorize it as

$$
\mathbf{X}=\mathbf{T T}^{T}
$$

where $\mathbf{T}$ is a lower triangular matrix with $t_{i i}>0, \forall i$. The Jacobian of the matrix transformation in Eq. (A.2) can be obtained from Theorem 1.28 in Mathai ${ }^{39}$ as

$$
d \mathbf{X}=2^{n} \prod_{i=1}^{n} t_{i i}^{n-i+1} d \mathbf{T}
$$


Due to the factorization in Eq. (A.2), we also have

$$
\begin{aligned}
& \operatorname{Trace}(\mathbf{X})=\operatorname{Trace}\left(\mathbf{T T}^{T}\right)=\sum_{j \leq i}^{n} t_{i j}^{2} \\
& \text { and } \quad|\mathbf{X}|=\left|\mathbf{T T}^{T}\right|=\left|\mathbf{T}^{2}\right|=\prod_{i=1}^{n} t_{i i}^{2} .
\end{aligned}
$$

Substituting Eqs. (A.3)-(A.5) in the integral (A.1) one has

$$
\begin{aligned}
\Gamma_{n}(a) & =2^{n} \iint_{\substack{-\infty<t_{i j}<\infty \\
t_{i i}>0}} \cdots \int_{n(n+1) \text { terms }} \exp \left(-\sum_{j \leq i}^{n} t_{i j}^{2}\right) \prod_{i=1}^{n}\left(t_{i i}^{2}\right)^{a-\frac{1}{2}(n+1)} \prod_{i=1}^{n} t_{i i}^{n-i+1} d t_{i j} \\
& =2^{n} \iint_{\substack{-\infty<t_{i j}<\infty \\
t_{i i}>0}} \cdots \int_{n(n+1) \text { terms }} \prod_{j \leq i}^{n} \exp \left(-t_{i j}^{2}\right) \prod_{i=1}^{n}\left(t_{i i}^{2}\right)^{a-\frac{1}{2}(i)} d t_{i j} .
\end{aligned}
$$

Separating the integrals involving the diagonal and off-diagonal terms and breaking $2^{n}$ into products of $n$ twos, Eq. (A.6) can be rewritten as

$$
\begin{aligned}
\Gamma_{n}(a)=\left[\prod_{j \leq i}^{n} \int_{-\infty<t_{i j}<\infty} \cdots \int_{n(n-1) \text { terms }} \exp \left(-t_{i j}^{2}\right) d t_{i j}\right] \\
\times\left[\prod_{i=1}^{n} 2 \int_{t_{i i}>0} \cdots \int_{n \text { terms }} \exp \left(-t_{i i}^{2}\right)\left(t_{i i}^{2}\right)^{a-\frac{1}{2}(i)} d t_{i i}\right] .
\end{aligned}
$$

Eq. (A.7) is now products of simple one dimensional integrals which can be evaluated easily ${ }^{43}$ to obtain

$$
\Gamma_{n}(a)=\pi^{\frac{1}{4} n(n-1)} \prod_{i=1}^{n} \Gamma\left[a-\frac{1}{2}(i-1)\right]
$$

The second term directly follows from the definition of the univariate gamma function. ${ }^{44}$ Next we introduce the concept of the matrix variate Laplace transform ${ }^{35}$ below:

Definition 5. Matrix variate Laplace transform: Let $f(\mathbf{X})$ be a function of a $n \times n$ symmetric positive definite matrix $\mathbf{X}$ and $\mathbf{Z}=\mathbf{Z}_{r}+\mathrm{i} \mathbf{Z}_{i}$, be a $n \times n$ symmetric complex matrix. Then the matrix variate Laplace transform $\mathcal{F}(\mathbf{Z})$ of $f(\mathbf{X})$ is defined as

$$
\mathcal{F}(\mathbf{Z})=\mathbb{L}\{f(\mathbf{X})\}=\int_{\mathbf{X}_{>0}} \operatorname{etr}\{-\mathbf{Z X}\} f(\mathbf{X}) d \mathbf{X}
$$

where the integral is assumed to absolutely convergent in the right half plane $\Re(\mathbf{Z})=\mathbf{Z}_{r}>0$.

We consider the following useful Laplace transform

$$
\mathbb{L}\left\{|\mathbf{X}|^{a-(n+1) / 2}\right\}=\int_{\mathbf{X}_{>0}} \operatorname{etr}\{-\mathbf{Z X}\}|\mathbf{X}|^{a-(n+1) / 2} d \mathbf{X}
$$

for $\Re(a)>\frac{1}{2}(n-1)$. Suppose $\mathbf{X}=\mathbf{Z}^{-1 / 2} \mathbf{Y} \mathbf{Z}^{-1 / 2}$ so that $d \mathbf{X}=|\mathbf{Z}|^{-\frac{1}{2}(n+1)} d \mathbf{Y}$ (see Chapter 1 in $\mathbf{M a t h a i}^{39}$ ). Substituting $\mathbf{X}$ into Eq. (A.10) we have

$$
\begin{aligned}
\mathbb{L}\left\{|\mathbf{X}|^{a-(n+1) / 2}\right\} & =\int_{\mathbf{Y}>0} \operatorname{etr}\{-\mathbf{Y}\}\left|\mathbf{Z}^{-1 / 2} \mathbf{Y} \mathbf{Z}^{-1 / 2}\right|^{a-(n+1) / 2}|\mathbf{Z}|^{-\frac{1}{2}(n+1)} d \mathbf{Y} \\
& =|\mathbf{Z}|^{-a} \int_{\mathbf{Y}>0} \operatorname{etr}\{-\mathbf{Y}\}|\mathbf{Y}|^{a-(n+1) / 2} d \mathbf{Y} .
\end{aligned}
$$

Using the the definition of the multivariate gamma function in Eq. (A.1), this equation can be written as

$$
\mathbb{L}\left\{|\mathbf{X}|^{a-(n+1) / 2}\right\}=\int_{\mathbf{X}>0} \operatorname{etr}\{-\mathbf{Z X}\}|\mathbf{X}|^{a-(n+1) / 2} d \mathbf{X}=|\mathbf{Z}|^{-a} \Gamma_{n}(a) .
$$

This expression is simply the matrix generalization of the well known scalar $(n=1)$ case (see reference ${ }^{45}$ for example) $\mathbb{L}\left\{t^{m-1}\right\}=\frac{\Gamma(m)}{s^{m}}$. Equation (A.12) turns out to be very useful as can be seen in the following section. 


\section{B. Proof of Theorem 1}

The main task is to obtain the expressions for the Lagrange multipliers $\lambda_{0} \in \mathbb{R}$ and $\boldsymbol{\Lambda}_{1} \in \mathbb{R}^{n \times n}$ appearing in Eq. (31). Substituting $p_{\mathbf{C}}(\mathbf{C})$ from Eq. (31) into the normalization condition in (23) we have

$$
\int_{\mathbf{C}>0} \exp \left\{-\lambda_{0}\right\} \operatorname{etr}\left\{-\boldsymbol{\Lambda}_{1} \mathbf{C}\right\} d \mathbf{C}=1 \quad \text { or } \quad \exp \left\{\lambda_{0}\right\}=\int_{\mathbf{C}>0} \operatorname{etr}\left\{-\boldsymbol{\Lambda}_{1} \mathbf{C}\right\} d \mathbf{C} .
$$

The last integral can be evaluated exactly in closed-form using the Laplace transform (A.12) by substituting $a=$ $\frac{1}{2}(n+1)$ as

$$
\exp \left\{\lambda_{0}\right\}=\left|\boldsymbol{\Lambda}_{1}\right|^{-(n+1) / 2} \Gamma_{n}\left(\frac{1}{2}(n+1)\right)=\left|\boldsymbol{\Lambda}_{1}\right|^{-r} \Gamma_{n}(r)
$$

where $r=\frac{1}{2}(n+1)$ as defined in Eq. (32). Substituting $\exp \left\{\lambda_{0}\right\}$ from Eq. (B.2) in the expressions of the pdf in Eq. (31) we have

$$
p_{\mathbf{C}}(\mathbf{C})=\left\{\Gamma_{n}(r)\right\}^{-1}\left|\boldsymbol{\Lambda}_{1}\right|^{r} \operatorname{etr}\left\{-\boldsymbol{\Lambda}_{1} \mathbf{C}\right\} .
$$

Now we need to obtain the matrix $\boldsymbol{\Lambda}_{1}$ from the second constraint equation (24). In order to avoid the direct evaluation of this integral, we will obtain the mean corresponding to the distribution in Eq. (B.3) using the characteristic function. The matrix variate characteristic function of $\mathbf{C}$ can be defined as

$$
\phi_{\mathbf{C}}(\boldsymbol{\Omega})=\mathrm{E}[\operatorname{etr}\{\mathrm{i} \boldsymbol{\Omega} \mathbf{C}\}]=\int_{\mathbf{C}>0} \operatorname{etr}\{\mathrm{i} \boldsymbol{\Omega} \mathbf{C}\} p_{\mathbf{C}}(\mathbf{C}) d \mathbf{C}
$$

where $\Omega$ is a symmetric matrix. Substituting the expression of the pdf from Eq. (B.3) into the preceding equation we have

$$
\phi_{\mathbf{C}}(\boldsymbol{\Omega})=\left\{\Gamma_{n}(r)\right\}^{-1}\left|\boldsymbol{\Lambda}_{1}\right|^{r} \int_{\mathbf{C}>0} \operatorname{etr}\left\{-\left(\boldsymbol{\Lambda}_{1}-\mathrm{i} \boldsymbol{\Omega}\right) \mathbf{C}\right\}|\mathbf{C}|^{r-\frac{1}{2}(n+1)} d \mathbf{C} .
$$

Again, this integral can be evaluated exactly in closed-form using the Laplace transform (A.12) by substituting $\mathbf{Z}=$ $\Lambda_{1}-\mathrm{i} \boldsymbol{\Omega}$ and $a=r$ as

$$
\phi_{\mathbf{C}}(\boldsymbol{\Omega})=\left|\boldsymbol{\Lambda}_{1}\right|^{r}\left|\boldsymbol{\Lambda}_{1}-\mathrm{i} \boldsymbol{\Omega}\right|^{-r}=\left|\mathbf{I}-\mathrm{i} \boldsymbol{\Omega} \boldsymbol{\Lambda}_{1}^{-1}\right|^{-r} .
$$

Therefore, the cumulant generating function

$$
\ln \phi_{\mathbf{C}}(\boldsymbol{\Omega})=-r \ln \left|\mathbf{I}-\mathrm{i} \boldsymbol{\Omega} \boldsymbol{\Lambda}_{1}^{-1}\right|=r\left(\mathrm{i} \boldsymbol{\Omega} \boldsymbol{\Lambda}_{1}^{-1}+\left[\mathrm{i} \boldsymbol{\Omega} \boldsymbol{\Lambda}_{1}^{-1}\right]^{2}+\cdots\right) .
$$

The mean of $\mathbf{C}$ can be obtained as

$$
\mathrm{E}[\mathbf{C}]=\left.\frac{\partial \ln \phi \mathbf{C}}{\partial(\mathrm{i} \boldsymbol{\Omega})}\right|_{\boldsymbol{\Omega}=\mathbf{O}}=r \boldsymbol{\Lambda}_{1}^{-1}
$$

Comparing this with Eq. (24) we have

$$
r \boldsymbol{\Lambda}_{1}^{-1}=\overline{\mathbf{C}} \quad \text { or } \quad \boldsymbol{\Lambda}_{1}=r \overline{\mathbf{C}}^{-1} .
$$

Equations (B.2) and (B.9) define both the unknown constants in the pdf of C. Substituting $\boldsymbol{\Lambda}_{1}$ in Eq. (B.3) we have

$$
p_{\mathbf{C}}(\mathbf{C})=\left\{\Gamma_{n}(r)\right\}^{-1}\left|r \overline{\mathbf{C}}^{-1}\right|^{r} \operatorname{etr}\left\{-r \overline{\mathbf{C}}^{-1} \mathbf{C}\right\}=r^{n r}\left\{\Gamma_{n}(r)\right\}^{-1}|\overline{\mathbf{C}}|^{-r} \text { etr }\left\{-r \overline{\mathbf{C}}^{-1} \mathbf{C}\right\}
$$

which proves the theorem.

To compare this pdf with the expression of the Wishart distribution in Eq. (19), substitute the expression of $r=\left(\frac{n+1}{2}\right)$ in Eq. (B.10) to obtain

$$
p_{\mathbf{C}}=\left(\frac{n+1}{2}\right)^{n\left(\frac{n+1}{2}\right)}\left\{\Gamma_{n}\left(\frac{n+1}{2}\right)\right\}^{-1}|\overline{\mathbf{C}}|^{-\left(\frac{n+1}{2}\right)} \operatorname{etr}\left\{-\left(\frac{n+1}{2}\right) \overline{\mathbf{C}}^{-1} \cdot \mathbf{C}\right\}
$$

This expression can be rearranged as

$$
p_{\mathbf{C}}=(2)^{\frac{-n(n+1)}{2}}\left\{\Gamma_{n}\left(\frac{n+1}{2}\right)\right\}^{-1}\left|\left(\frac{\overline{\mathbf{C}}}{n+1}\right)\right|^{-\left(\frac{n+1}{2}\right)}|\mathbf{C}|^{\frac{1}{2}\{(n+1)-(n+1)\}} \operatorname{etr}\left\{-\frac{1}{2}\left(\frac{\overline{\mathbf{C}}}{n+1}\right)^{-1} \mathbf{C}\right\} .
$$

Comparing Eq. (B.12) with the Wishart distribution in Eq. (19) it can be observed that $\mathbf{C}$ has the Wishart distribution with parameters $p=n+1$ and $\boldsymbol{\Sigma}=\overline{\mathbf{C}} /(n+1)$. 